\title{
Prácticas docentes, clima social, seguridad escolar y violencia entre estudiantes
}

Teaching Practices, Social Climate, School Safety and Student Violence

Les pratiques des enseignants, le clima social, la sécurité scolaire et la violence parmi les étudiants

Práticas docentes, clima social, segurança escolar e violência entre estudantes

Fecha de recepción: 6 DE MAYO DE 2016/Fecha de aceptación: 12 DE SEPTIEMBRE DE 2017/Fecha de disponibilidad en línea: 15 DE ABRIL DE 2018

Encuentre este artículo en http://magisinvestigacioneducacion.javeriana.edu.co/ Instituto TECnOLÓgico de SONORA

JEsÚs TÁNORI-QUINTANA Instituto TeCnológico de SONORA SONORA, MÉXICO jesus.tanori@itson.edu.mx

Teresa IVEth SOtelo-Quiñonez instituto Tecnológico de Sonora SONORA, MÉXICO

\section{Resumen}

El contexto escolar previene el acoso escolar. El estudio analizó las relaciones entre las prácticas docentes de intervención directa, la seguridad, el clima escolar y el acoso entre estudiantes. La muestra se compone de 291 estudiantes con edades entre 9 y 13 años $(M=10.94, D E=2.77)$ de 17 escuelas primarias de un estado de México. Los resultados de la regresión lineal sugieren que las prácticas de intervención directa, la seguridad y un clima escolar positivo disminuyen el acoso. Se concluye que las variables involucradas en el estudio previenen el acoso. Finalmente, se discuten implicaciones de los hallazgos.

\section{Palabras claves}

Violencia; escuela; acoso; papel del docente 
Keywords

Violence; school; bullying; teacher's role

\section{Abstract}

The school context prevents bullying. The study analyzed the relationship between teaching practices of direct intervention, safety, school climate and harassment among students. The sample consists of 291 students aged between 9 and 13 years old $(M=10.94, S D=2.77)$ from 17 elementary schools in a state of Mexico. Results of the linear regression suggest that direct intervention practices, safety and a positive school climate reduce harassment. We concluded that the variables involved in the study prevent harassment. Finally, we discuss the implications of the findings.

\section{Mots clés}

Violence ; école ; harcèlement scolaire ; rôle de l'enseignant

\section{Résumé}

Le contexte scolaire avertit le harcèlement scolaire. L'étude analyse les rapports entre les pratiques des enseignants d'intervention directe, la sécurité, le climat scolaire et le harcèlement parmi les étudiants. L'échantillon est composé de 291 étudiants âgés entre les 9 et 13 ans $(M=10.94, D E$ $=2.77$ ) de 17 écoles primaires d'un état du Mexique. Les résultats de la régression linéaire montrent que les pratiques d'intervention directe, la sécurité et un climat scolaire positif diminuent le harcèlement. On conclut que les variables inclus dans l'étude préviennent le harcèlement. Enfin on discute les implications des résultats.
Palavras-chave

Violência; escola; assédio; papel do docente

\section{Resumo}

O contexto escolar previne o assédio escolar. O estudo analisou as relações entre as práticas docentes de intervenção direta, a segurança, o clima escolar e o assédio entre estudantes. A mostra compõe-se de 291 estudantes com idades entre 9 e 13 anos $(\mathrm{M}=10.94, \mathrm{DE}=2.77)$ de 17 escolas primárias de um estado do México. Os resultados da regressão linear sugerem que as práticas de intervenção direta, a segurança e um clima escolar positivo diminuem o assédio. Conclui-se que as variáveis envolvidas no estudo previnem o assédio. Finalmente, discutem-se implicações dos resultados. 


\section{Introducción}

En la Reforma Integral de la Educación Básica (RIEB) realizada en México (México, 2011, Acuerdo 592) se establece que las escuelas deben desarrollar valores y habilidades socioemocionales en los estudiantes para afrontar de forma asertiva situaciones sociales, convivir de forma armónica e integrarse al mundo social (García-Cabrero, 2012). Los objetivos antes expuestos no se cumplen en su totalidad, ya que existe evidencia de que alrededor del 25,0\% de los alumnos de educación básica participan como víctimas $y / 0$ agresores en situaciones de violencia entre estudiantes, y un 47,2\% refiere estar presente cuando ocurren las agresiones (Aguilera-García, Muñoz-Abundez \& Orozco-Martínez, 2007; Haro-Solís \& García-Cabrero, 2014; Haro-Solís, García-Cabrero \& Reidl-Martínez, 2013; Muñoz-Abundez, 2008; Valdés-Cuervo, Bautista-Hernández, Vera-Noriega \& Herrera-Mateos, 2013).

El acoso entre pares influye de forma negativa en el ambiente de aprendizaje y en el propósito de las instituciones educativas de formar ciudadanos que convivan en el marco del respeto por los derechos de otras personas. En particular, se ven afectados los estudiantes involucrados de manera directa o indirecta como víctimas o agresores en situaciones de acoso. En las víctimas se constata mayor prevalencia de problemas de ansiedad, depresión, dificultades en las relaciones interpersonales y conductas autodestructivas (Meltzer, Vostanis, Ford, Bebbington \& Dennis, 2011; O'Brennan, Bradshaw \& Sawyer, 2009). Por su parte, en los agresores se reportan consumo de sustancias, dificultades de ajuste psicosocial y conductas antisociales que perduran en la juventud (Kokkinos \& Panayiotou, 2004; Olweus, 2011). Incluso en los estudiantes espectadores regulares de comportamientos agresivos hacia sus pares se refieren dificultades emocionales y desconexión moral ante la agresión (Rigby, 2003; Thomas \& Bierman, 2006).

El acoso entre estudiantes requiere abordarse desde una perspectiva ecológica para su prevención. Este problema se asocia a características tanto de los estudiantes como de sus contextos familiares, escolares y comunitarios (Álvarez-García, García \& Núñez, 2015; Swearer \& Hymel, 2015). Dada la complejidad del acoso escolar hay acuerdo en que, para su prevención, son necesarias intervenciones integrales en las que participen los diversos actores involucrados en el proceso educativo (Kilian, Fish \& Maniago, 2007; Whitted \& Dupper, 2005).

Las acciones de las escuelas para prevenir el acoso escolar se relacionan con una menor prevalencia de esta problemática (American Educational Research Association, AERA, 2013; Swearer, Espegale, Koenig, Berry, Collins \& Lembeck, 2012). A pesar de la evidencia sobre la importancia de los factores escolares para la prevención de la violencia escolar, aún es escasa la investigación realizada en México en la temática (Carrillo-Navarro, Prieto-Quezada \& Jiménez-Mora, 2013). Se han constatado los efectos de las prácticas docentes de intervención directa en situaciones de agresión (Kochenderfer-Ladd \& Pelletier, 2008; Yoon \& Kerber, 2003), el clima social y las acciones de seguridad escolar en el acoso entre estudiantes (Cava, Musitu, Buelga \& Murgui, 2010; Cerezo-Ramírez \& Ato-García, 2010).

Las prácticas docentes involucran acciones pedagógicas realizadas por los profesores con los estudiantes y otros actores involucrados en la educación (Akalin \& Sucuoglu, 2015; García-Cabrero, Loredo-Enríquez \& Carranza-Peña, 2008). Estas prácticas contribuyen a prevenir o reforzar las conductas agresivas entre estudiantes (Fekkes, Pijpers \& Verloove-Vanhorick, 2005; Kochenderfer-Ladd \& Pelletier, 2008). Se constata que cuando
Descripción del artículo | Article description | Description de l'article | Artigo descrição

Este artículo de investigación, derivado del proyecto Factores asociados al bullying en escuelas primarias del sur de Sonora. Un enfoque multinivel, analiza la relación de variables relativas al contexto escolar (prácticas docentes, clima social y acciones de seguridad escolar) con la problemática de la agresión entre estudiantes en educación básica. Se concluye que las características del contexto escolar se relacionan de forma importante con la problemática de la violencia escolar. 
los profesores detienen las agresiones, apoyan a las víctimas y/o sancionan a los agresores previenen el acoso (Alfaro-Molina, Kenton-Paniagua \& Leiva-Díaz, 2010; Bauman \& Del Río, 2005; Wei, Williams, Chen \& Chang, 2010). Además, estas prácticas favorecen que los estudiantes experimenten confianza en el compromiso y las habilidades de los docentes para detener el acoso (Hanish \& Guerra, 2000; Harris, Petrie \& Willoughby, 2002; Houndoumadi \& Pateraki, 2001).

El clima social escolar se refiere a la calidad de la vida escolar (Cohen, McCabe, Michelli \& Pickeral, 2009). Un elemento crítico del mismo, asociado con la prevención del acoso escolar, es la relación entre docentes y estudiantes (Furlong, Greif, Bates, Whipple y Jiménez, 2005; Trianes-Torres, BlancaMena, Morena-Fernández, 2006; Infante-Cañete \& Raya, 2006). Un clima social escolar positivo reduce el estrés, los problemas socioemocionales y el acoso entre pares (Bosworth \& Judkins, 2014; Cerezo-Ramírez \& Ato-García, 2010; Sangsue \& Vorpe, 2004). También promueve en los estudiantes el compromiso con las normas escolares y el desarrollo de competencias socioemocionales para la solución pacífica de los conflictos (Mehta, Cornell, Fan \& Gregory, 2013; Wang, Berry \& Swearer, 2013).

Por su parte, la seguridad escolar implica acciones en las escuelas para prevenir el acoso y garantizar el bienestar de los estudiantes (Shelton, Owens \& Song, 2009). De acuerdo con Amanda B. Nickerson y Matthew P. Martens (2008), la seguridad escolar incluye acciones restrictivas $y / 0$ educativas en las escuelas. Las acciones educativas, centro de interés del estudio, tienen como fin fortalecer la vivencia de seguridad psicológica de los estudiantes en las escuelas (Nickerson \& Spears, 2007). Estas acciones incluyen la mediación del docente en conflictos, orientación educativa, supervisión de los espacios escolares, socialización del código de disciplina y de los procedimientos para resolver situaciones de violencia. La literatura constata que las acciones de seguridad educativas disminuyen las conductas agresivas, favorecen el compromiso con las normas escolares y las conductas prosociales de los estudiantes hacia sus pares (Osher, Dwyer, Jimerson \& Brown, 2012; Stephens, 2011).

Si bien hay evidencia empírica acerca del rol de la escuela en la prevención del acoso escolar, en la revisión de la literatura, se aprecian limitaciones en el estudio de la temática. En primer lugar, no se identificaron estudios que analicen de forma conjunta la influencia de las prácticas docentes de intervención directa en las situaciones de violencia, la seguridad escolar y el clima social de la escuela en el acoso entre estudiantes. En segundo lugar, en México se hallaron escasas publicaciones acerca de la relación del contexto escolar, y, en particular, de las variables analizadas en el acoso.
Atendiendo a esto, el presente estudio se propuso analizar la relación entre las prácticas docentes de intervención directa en situaciones de violencia, las acciones educativas de seguridad escolar y el clima social de la escuela con el acoso entre estudiantes. Se espera que las prácticas docentes de intervención directa, las acciones educativas de seguridad escolar y el clima escolar positivo se relacionen negativamente con la frecuencia de acoso entre estudiantes.

\section{Método}

\section{Participantes}

Como criterios de inclusión se consideró que las escuelas se ubicaran en distintas zonas escolares del estado de Sonora, y que estuvieran dispuestas a colaborar en el estudio. Se seleccionaron, de forma no probabilística, 291 estudiantes con edades comprendidas entre 9 y 13 años $(M=10.94, D E=2.77$ años) de 17 escuelas primarias públicas urbanas. La muestra se integró por 166 (57.00\%) niños y 125 (43.00\%) niñas. Del total de los participantes, 63 (21.32\%) cursaban el cuarto, 135 (46.41\%) el quinto y 93 (31.97\%) el sexto grado.

\section{Instrumentos}

Acoso entre estudiantes. Se utilizó la escala de autorreporte, desarrollada por Ángel Alberto ValdésCuervo y Ernesto Alonso Carlos-Martínez (2014). En esta, los estudiantes refieren la frecuencia de acoso por parte de compañeros de escuela durante el último mes (ejemplo: me amenazan, no permiten que participe en juegos). El instrumento se compone de nueve ítems que se contestan con una escala tipo Likert con cinco opciones de respuesta 0 (nunca), 1 (casi nunca 0-2 agresiones), 2 (a veces 3-5 agresiones), 3 (casi siempre 5-7 agresiones) y 4 (siempre más de 7 agresiones). Mediante un análisis factorial confirmatorio con el método de máxima verosimilitud, se confirmó la sustentabilidad de un modelo de medición unidimensional del constructo $\left(X^{2}=15.31, g l=8, p=.36 ; X^{2} / g l\right.$ $=1.78 ; \mathrm{CFI}=.97 ; \mathrm{AGFI}=.95 ; \mathrm{SRMR}=.05 ; \mathrm{RMSEA}=$ .04, IC 90 [.02 - .07]). La fiabilidad medida con el alfa de Cronbach fue de .85 .

Prácticas docentes de intervención directa en la violencia. Se midió con la subescala del instrumento Prácticas Docentes No Permisivas de la Violencia (PDNP) (Valdés-Cuervo, Martínez-Ferrer \& Carlos-Martínez, 2018). Esta subescala mide conductas del docente dirigidas a interrumpir la agresión, sancionar al agresor y proteger a la víctima. Consta de cuatro ítems (ejemplo: sancionan al agresor de forma adecuada, apoyan a las víctimas para detener al agresor). Se responde en un formato tipo Likert con cinco opciones de respuesta 
que oscilan desde 0 (nunca), 1 (casi nunca), 2 (a veces), 3 (frecuentemente) y 4 (siempre). Mediante un análisis factorial confirmatorio con ecuaciones estructurales se estableció el ajuste del modelo medición unidimensional de medición del constructo $\left(X^{2}=9.87, p=.27 ; X^{2} / g l=1.23 ;\right.$ SRMR $=.06$; $\mathrm{AGFI}=.97 ; \mathrm{CFI}=.99 ; \mathrm{RMSEA}=.03, \mathrm{IC} 90[.01-.06])$. La fiabilidad medida con el alfa de Cronbach fue de .79 .

Clima social escolar. Se utilizó el cuestionario desarrollado por María Victoria Trianes-Torres, María José Blanca-Mena, María Luisa de la MorenaFernández, Lidia Infante-Cañete y S. Raya (2006), que mide la calidad de las relaciones sociales del estudiante en la escuela. La escala se compone de nueve ítems que miden la percepción del estudiante con respecto al apoyo, la confianza y el respeto en sus relaciones con los docentes (ejemplo: mi escuela es un lugar seguro, los profesores son amables con los estudiantes). Los reactivos se responden mediante una escala tipo Likert con cinco opciones de respuesta 0 (nunca), 1 (casi nunca), 2 (a veces), 3 (frecuentemente) y 4 (siempre). Mediante el cálculo de un análisis factorial confirmatorio con ecuaciones estructurales se obtuvieron valores de $X^{2}=58.81, g l=29$, $p=.001 ; X^{2} / g l=2.10 ;$ SMRM $=.05 ; \mathrm{AGFI}=.92 ; \mathrm{CFI}=.97 ; \mathrm{RMSEA}=$ .05 , IC 90 [.03 - .08]). Los resultados sugieren que el modelo de medición se ajusta a los datos, ya que si bien el valor de probabilidad asociada a $X^{2}$ fue significativa - lo cual se explica por la vulnerabilidad de la prueba al tamaño de muestra - los valores de los otros índices de ajuste resultaron aceptables (Blunch, 2013; Byrne, 2010). La fiabilidad medida con el alfa de Cronbach fue de 89.

Seguridad escolar. Se desarrolló ex profeso para el estudio con base en el análisis de la literatura en la temática (Meyer-Adams \& Conner, 2008; Nickerson \& Martens, 2008). Este midió, desde la perspectiva de los estudiantes, la frecuencia de acciones educativas de seguridad en las escuelas. Consta de siete ítems (ejemplo: los docentes discuten con los estudiantes las normas de disciplina de la escuela, los docentes u otros adultos supervisan los salones de clases y otros espacios de la escuela). Para su respuesta se utilizó una escala tipo Likert 0 (nunca), 1 (casi nunca), 2 (a veces), 3 (frecuentemente) y 4 (siempre). Mediante un análisis factorial confirmatorio, se validó la estructura de medición unidimensional del constructo $\left(X^{2}=17.98, p=.16 ; X^{2} / g l=1.38 ;\right.$ SRMR $=.05$ $\mathrm{AGFI}=.97 ; \mathrm{CFI}=.99 ; \mathrm{RMSEA}=.03, \mathrm{IC} 90[.01-.06])$. La fiabilidad medida con el alfa de Cronbach fue de .81 .

\section{Procedimiento}

Se informó a las autoridades de las escuelas acerca del objetivo de la investigación y se solicitó su autorización para acceder a los salones de clase. Posteriormente, se envió con los estudiantes una carta a los progenitores que explicaba el propósito de la investigación y se les pedía que firmen un consentimiento informado en el que les otorgaban a los hijos el permiso para contestar los cuestionarios. A los estudiantes cuyos padres firmaron la carta de consentimiento informado, se les invitó a participar de forma voluntaria en el estudio garantizándoles confidencialidad de sus respuestas.

La administración de los instrumentos se realizó en los salones de clase por parte de dos integrantes del equipo de investigación debidamente entrenados. Para garantizar el beneficio de los involucrados se entregó un reporte de resultados con recomendaciones, tanto a las autoridades de las escuelas como a los responsables de las asociaciones de padres. 
Análisis estadístico

Los datos perdidos se trataron con el método de imputación por regresión. En un primer momento, con apoyo del SPSS v. 23, se calcularon las medias, las desviaciones estándar y las correlaciones entre las variables. En segundo lugar, se realizó el cálculo de un modelo de regresión lineal múltiple jerárquica. Por último, se analizó el tamaño del efecto con el software G*Power v. 3.1.6.

\section{Resultados}

Los valores de los coeficientes de correlación de Pearson sugieren que los reportes de acoso por pares se correlacionan de forma negativa con prácticas docentes de intervención directa en situaciones de violencia, acciones educativas de seguridad escolar y un clima escolar positivo (tabla 1).

Tabla 1

Media, desviación estándar y correlación entre acoso escolar y las variables predictoras prácticas docentes, clima social escolar y seguridad escolar

\begin{tabular}{l|c|c|c|c|c|c}
\hline Variables & $\mathbf{M}$ & $\mathbf{D E}$ & $\mathbf{1}$ & $\mathbf{2}$ & $\mathbf{3}$ & $\mathbf{4}$ \\
\hline Acoso entre estudiantes & 2.41 & .43 & - & & & \\
\hline Variables predictoras & & & & & & \\
\hline Prácticas docentes de intervención directa & 1.83 & .52 & $.47^{* * *}$ & - & & \\
\hline Clima social escolar & 3.12 & .48 & $-.39^{* * *}$ & $.22^{* * *}$ & - & \\
\hline Seguridad escolar & 3.17 & .68 & $-.34^{* * *}$ & $.19^{* * *}$ & $.25^{* * *}$ & - \\
\hline
\end{tabular}

${ }^{*} p<.05 .{ }^{* *} p<01 .{ }^{* * *} p<.001$.

Fuente: elaboración propia

Los resultados sugieren que las prácticas docentes de intervención directa, el clima social escolar y las acciones de seguridad escolar explican una parte significativa del acoso entre estudiantes de primaria $\left(R^{2}=.39\right)$. El tamaño del efecto es grande $\left(f^{2}=.64\right)$, lo que indica el valor práctico de las relaciones encontradas.

Del análisis de los coeficientes $B$ se infiere que todas las variables estudiadas se relacionan de forma negativa con el acoso. Esto implica que las prácticas docentes de intervención directa en situaciones de violencia, la calidad del clima social de la escuela y las acciones educativas de seguridad escolar disminuyen la frecuencia de acoso entre estudiantes (tabla 2).

Tabla 2

Resultados de la regresión jerárquica para las que predicen el acoso por pares ( $N=291)$

\begin{tabular}{l|c|c|c|c|c}
\hline Paso y variables predictoras & $\boldsymbol{B}$ & $\boldsymbol{S E} \boldsymbol{B}$ & $\boldsymbol{B}$ & $\boldsymbol{R}^{\mathbf{2}}$ & $\boldsymbol{\Delta}$ \\
\hline $\begin{array}{l}\text { Paso 1 } \\
\text { Prácticas docentes de intervención directa }\end{array}$ & 0.40 & 0.49 & $-.47^{* * *}$ & $.22^{* * *}$ & \\
\hline $\begin{array}{l}\text { Paso 2 } \\
\text { Seguridad escolar }\end{array}$ & -0.18 & 0.25 & $-.41^{* * *}$ & $.32^{* * *}$ & $.10^{* *}$ \\
\hline $\begin{array}{l}\text { Paso 3 } \\
\text { Clima social escolar }\end{array}$ & -0.18 & 0.29 & $-.35^{* *}$ & $.39^{* * *}$ & $.07^{* *}$ \\
\hline \hline
\end{tabular}

${ }^{*} p<.05 .{ }^{* *} p<01 .{ }^{* * *} p<.001$.

Fuente: elaboración propia 


\section{Discusión}

El estudio indaga la relación entre prácticas docentes de intervención directa en situaciones de violencia, las acciones educativas de seguridad escolar y el clima social de la escuela con el acoso entre estudiantes. Se esperaba que las prácticas docentes de intervención directa, las acciones educativas de seguridad escolar y un clima social positivo en la escuela se relacionaran negativamente con la frecuencia de acoso entre estudiantes. Los resultados confirman las hipótesis propuestas en el sentido de que las prácticas docentes de intervención directa, la seguridad escolar y el clima social de la escuela se relacionan negativamente con el acoso entre estudiantes.

Los resultados son similares a lo reportado en la literatura respecto a que la intervención directa del docente en situaciones de violencia disminuye la frecuencia del acoso entre estudiantes (Kilian, Fish \& Maniago, 2007; Valdés-Cuervo, Martínez-Ferrer \& Carlos-Martínez, 2018; Whitted \& Dupper, 2005). En particular, se constató que las prácticas docentes que implican detener la agresión, sancionar al agresor y/o proteger son acciones pertinentes para prevenir el acoso (Cava, Musitu, Buelga \& Murgui, 2010; Collie, Shapka \& Perry, 2011; Kochenderfer-Ladd \& Pelletier, 2008).

También los hallazgos del estudio, de forma congruente con la literatura, sugieren que las acciones de seguridad escolar que involucran la creación de estructura (normas de convivencia y mecanismos para atender los casos de violencia) y el apoyo a los estudiantes (orientación y mediación en conflictos) disminuyen el acoso entre estudiantes (Nickerson \& Martens, 2008; Shelton, Owens \& Song, 2009). Esto muestra la necesidad de programas de prevención del acoso enfocados en el desarrollo de acciones educativas dirigidas a fortalecer la percepción de seguridad psicológica de los estudiantes (Boulton, 2014; Flaspohler, Elfstrom, Vanderzee, Sink \& Birchmeier, 2009; Kahn, Jones \& Wieland, 2012).

Se encontró, además, que un clima social escolar positivo se relaciona con menor frecuencia de acoso escolar. Este resultado es consistente con lo reportado en la literatura $y$, en este sentido, reafirma el valor de este constructo en la prevención de la violencia entre estudiantes (Cornell \& Bradshaw, 2015; Loukas \& Murphy, 2007). En particular, la importancia de las relaciones cercanas entre docentes y estudiantes en la percepción de un clima escolar de calidad, y el impacto de esta forma de interacción en la disminución del acoso escolar.

En síntesis, los resultados muestran el valor de los docentes y el ambiente escolar en la prevención del acoso entre estudiantes. Se constata que los programas de prevención de la violencia deben incluir acciones de capacitación a los profesores para intervenir efectivamente en situaciones de violencia, y desarrollar relaciones cercanas con los estudiantes. Además, es esencial que como parte de la prevención se incorporen en la gestión escolar medidas encaminadas a fortalecer la seguridad psicológica de los estudiantes. También Ilama la atención que los estudiantes reportan pocas prácticas de intervención directa de los profesores en situaciones de violencia. Este hallazgo que es consistente con lo reportado en estudios anteriores (Burger, Strohmeier, Spröber, Bauman \& Rigby, 2015; Kahn, Jones \& Wieland, 2012), sugiere que algunos docentes no se involucran activamente en la prevención del acoso. Este hallazgo sugiere que es necesario sensibilizar y capacitar a los docentes para que asuman su rol en la prevención del acoso escolar.

Se puede hipotetizar, por otro lado, que las prácticas docentes de intervención directa, la seguridad escolar y el clima escolar positivo previenen el acoso por sus efectos en la conducta de los estudiantes. Disminuyen las ganancias instrumentales y socioafectivas que obtienen los agresores por su comportamiento (Caravita, Di Blasio \& Salmivalli, 2009; Hymel \& Swearer, 2015; Salmivalli \& Nieminen, 2002). Aumenta el apoyo que reciben las víctimas, lo cual favorece que afronten de manera directa y/o busquen ayuda en los adultos de la escuela en situaciones de acoso (MadridLópez, Valdés-Cuervo \& Vera-Noriega, 2015; Nation, Vieno, Perkins \& Santinello, 2008). Al fomentar un ambiente social de no tolerancia hacia la violencia, se promueve que los espectadores desarrollen conductas prosociales hacia las víctimas (Jungert, Piroddi \& Thornberg, 2016; Salmivalli \& Voeten, 2004).

Si bien el presente estudio aporta información de valor para la prevención del acoso escolar, los resultados presentan limitaciones, que implican que deban interpretarse con cierta cautela. En primer lugar, el diseño del estudio de tipo transversal no permite establecer relaciones causales entre las variables. Se sugiere en futuras investigaciones realizar diseños experimentales y longitudinales que permitan precisar estas relaciones. En segundo lugar, la muestra se compone de estudiantes de una zona geográfica específica de México, lo que dificulta la generalización de los hallazgos a otras regiones del país. En tercer lugar, puede haber otras variables del contexto escolar, además de las analizadas, que influyan en el acoso entre estudiantes. Al respecto, se sugiere realizar otros estudios que indaguen otras variables del docente, como la empatía hacia las víctimas y de la escuela, como la percepción de autoeficacia grupal para detener el acoso. 


\section{Sobre los autores}

Ángel Alberto Valdés-Cuervo es doctor en ciencias. Profesor investigador titular C, Departamento de Educación, Instituto Tecnológico de Sonora. Miembro del Consejo Mexicano de Investigación Educativa y Sistema Nacional de Investigadores Nivel I. Sus líneas de investigación versan sobre violencia escolar, familia-escuela, aptitudes sobresalientes y educación superior.

Jesús Tánori-Quintana es doctor en ciencias sociales. Profesor investigador auxiliar, Departamento de Educación, Instituto Tecnológico de Sonora. Miembro del Sistema Nacional de Investigadores, Nivel Candidato. Sus líneas de investigación versan sobre bienestar y violencia escolar.

Teresa Iveth Sotelo-Quiñonez es doctora en ciencias sociales. Profesora investigadora auxiliar, Departamento de Psicología, Instituto Tecnológico de Sonora. Miembro del Sistema Nacional de Investigadores, Nivel Candidato. Sus líneas de investigación versan sobre bienestar en cuidadores y violencia en el noviazgo.

José Alán Ochoa-Arreola es licenciado en psicología. Estudiante en la Maestría en Investigación Educativa en el Instituto Tecnológico de Sonora. Es colaborador del cuerpo académico de Procesos Educativos, donde se investiga sobre violencia escolar.

\section{Referencias}

Aguilera-García, María Antonieta; Muñoz-Abundez, Gustavo \& OrozcoMartínez, Adriana (2007). Disciplina, violencia y consumo de sustancias nocivas a la salud en escuelas primarias y secundarias mexicanas. Ciudad de México: Instituto Nacional para la Evaluación de la Educación, INEE. Disponible en: http://www.prevenciondelaviolencia.org/ system/files/recursos/35_disciplina_violencia_y_consumo.pdf

Ahmed, Eliza (2001). Shame Management: Regulating Bullying. En Eliza Ahmed, Nathan Harris, John Braithwaite \& Valerie Braithwaite (eds.). Shame Management through Reintegration, 221-314. Cambridge, United Kingdom: Cambridge University Press. Disponible en: http:// johnbraithwaite.com/wp-content/uploads/2016/06/Shame-Mana gement-through-Reint.pdf

Akalin, Selma \& Sucuoglu, Bulbin (2015). Effects of Classroom Management Intervention Based on Teacher Training and Performance Feedback on Outcomes of Teacher-Student Dyads in Inclusive Classrooms. Educational Sciences: Theory \& Practice, 15 (3), 739-758. http://dx.doi. org/10.12738/estp.2015.3.2543. Disponible en: http://www.estp. com.tr/wp-content/uploads/2015/05/ESTPApril2015_739_758.pdf

Alfaro-Molina, Ivannia; Kenton-Paniagua, Karen \& Leiva-Díaz, Viriam (2010). Conocimientos y percepciones del profesorado sobre violencia en los centros educativos públicos. Enfermería Actual en Costa Rica, 18, 1-10. Disponible en: www.redalyc.org/articulo.oa?id=44817860001

Álvarez-García, David; García, Trinidad \& Núñez, José Carlos (2015). Predictors of School Bullying Perpetration in Adolescence: a Systematic Review. Aggression and Violent Behavior, 23, 126-136. http://dx.doi. org/10.1016/j.avb.2015.05.007

American Educational Research Association, AERA (2013). Prevention of Bullying in Schools, Colleges, and Universities. Research Report and Recommendations. Washington, DC: AERA. Disponible en: https:// www.aera.net/Portals/38/docs/News\%20Release/Prevention\%20 of\%20Bullying\%20in\%20Schools,\%20Colleges\%20and\%20Univer sities.pdf 
Bauman, Sheri \& Del Río, Adrienne (2005). Knowledge and Beliefs about Bullying in Schools Comparing Pre-Service Teachers in the United States and the United Kingdom. School Psychology International, 26 (4), 428-442. DOI 10.1177/ 0143034305059019

Blunch, Niels J. (2013). Introduction to Structural Equation Modeling Using IBM SPSS Statistics and AMOS ( $2^{\text {nd }}$ ed.). Thousand Oaks, California: Sage.

Bosworth, Kris \& Judkins, Maryann (2014). Tapping into the Power of School Climate to Prevent BuIlying: One Application of School Wide Positive Behavior Interventions and Supports. Theory Into Practice, 53 (4), 300-307. http://dx.doi.org /10.1080/00405841.2014.947224

Boulton, Michael J. (2014). Teachers' Self-Efficacy, Perceived Effectiveness Beliefs, and Reported Use of Cognitive-Behavioral Approaches to Bullying among Pupils: Effects of In-Service Training with the I DECIDE Program. Behavior Therapy, 45 (3), 328-343. http://dx.doi.org/10.1016/j. beth.2013.12.004

Burger, Christoph; Strohmeier, Dagmar; Spröber, Nina; Bauman, Sheri \& Rigby, Ken (2015). How Teachers Respond to School Bullying: An Examination of Self-Reported Intervention Strategy Use, Moderators, and Concurrent Use Multiple Strategies. Teaching and Teacher Education, 51, 191-202. http://dx.doi.org/10.1016/j.tate.2015.07.004

Byrne, Barbara M. (2010). Structural Equation Modeling with AMOS. Basic concepts, applications, and programming ( $2^{\text {nd }}$ ed.). New York, New York: Routledge Taylor \& Francis Group.

Caravita, Simona C. S.; Blasio, Paola di \& Salmivalli, Christina (2009). Unique and Interactive Effects of Empathy and Social Status on Involvement in BuIlying. Social Development, 18 (1), 140-163. http:// dx.doi.org/10.1111/j.1467-9507.2008.00465.x

Carrillo-Navarro, José Claudio; Prieto-Quezada, María Teresa \& Jiménez-Mora, José (2013). Bullying, violencia entre pares en escuelas de México. En Alfredo Furlán-Malamud \& Terry Carol SpitzerSchwartz (eds.). Convivencia, disciplina y violencia en las escuelas 2002-2011, 223-260. México: Asociación Nacional de Universidades e Instituciones de Educación Superior en México, Consejo Mexicano de Investigación Educativa, ANUIES/ COMIE.

Cava, María Jesús; Musitu, Gonzalo; Buelga, Sofia \& Murgui, Sergio (2010). The Relationships of Family and Classroom Environments with Peer Relational Victimization: An Analysis of their Gender Differences. The Spanish Journal of Psychology, 13 (1), 156-165.
Cerezo-Ramírez, Fuensanta \& Ato-García, Manuel (2010). Social Status, Classroom Climate and BuIlying among Adolescent Pupils. Anales de Psicología, 26 (1), 137-144. Disponible en: http://re vistas.um.es/analesps/article/view/92131/88721

Cohen, Jonathan; McCabe, Elizabeth M.; Michelli, Nichollas M. \& Pickeral, Terry (2009). School Climate: Research, Policy, Practice and Teacher Education. Teacher College Record, 111 (1), 180-213. Disponible en: http://ww.ijvs.org/files/Publica tions/School-Climate.pdf

Collie, Rebecca J.; Shapka, Jennifer D. \& Perry, Nancy E. (2011). Predicting Teacher Commitment: the Impact of School Climate and Social-Emotional Learning. Psychology in the Schools, 48 (10), 1034-1048. http://dx.doi.org/10.1002/ pits.20611

Cornell, Dewey \& Bradshaw, Catherine P. (2015). From a Culture of Bullying to a Climate of Support: The Evolution of Bullying Prevention and Research. School Psychology Review, 44 (4), 499-503. http://dx.doi.org/10.17105/spr-15-0127.1

Ellis, Alicia A. \& Shute, Rosalyn (2007). Teacher Responses to Bullying in Relation to Moral Orientation and Seriousness of Bullying. British Journal of Educational Psychology, 77 (3), 649-663. http://dx.doi.org/10.1348/000709906X163405

Fekkes, Minne; Pijpers, Frans I. M. \& Verloove-Vanhorick, S. Pauline (2005). Bullying: Who Does What, When and Where? Involvement of Children, Teachers and Parents in Bullying Behavior. Health Education Research, 20 (1), 81-91. http://dx.doi. org/10.1093/her/cyg100. Disponible en: https:// academic.oup.com/her/article/20/1/81/632611

Flaspohler, Paul D.; Elfstrom, Jennifer L.; Vanderzee, Karin L.; Sink, Holli E. \& Birchmeier, Zachary (2009). Stand by Me: The Effects of Peer and Teacher Support in Mitigating the Impact of Bullying on Quality of Life. Psychology in the Schools, 46 (7), 636-649. http://dx.doi.org/10.1002/pits.20404

Furlong, Michael J.; Greif, Jennifer L.; Bates, Michael P.; Whipple, Angela D.; Jiménez, Terese C. \& Morrison, Richard (2005). Development of the California School Climate and Safety Survey-Short Form. Psychology in the School, 42 (2), 137-149. http://dx.doi.org/10.1002/pits.20053

García-Cabrero, Benilde (2012). Consolidar las reformas de la educación básica en México. Una asignatura pendiente. Perfiles Educativos, 34 (número especial), 47-56. Disponible en: http:// www.redalyc.org/articulo.oa?id=13229959005

García-Cabrero, Benilde; Loredo-Enríquez, Javier \& Carranza-Peña, Guadalupe (2008). Análisis de la práctica educativa de los docentes: pensamiento, interacción y reflexión. Revista Electrónica de Investigación Educativa, número especial, 1-15. 
Disponible en: https://redie.uabc.mx/redie/arti cle/view/200/345

Hanish, Laura D. \& Guerra, Nancy G. (2000). Children Who Get Victimized at School: What Is Known? What Can Be Done? Professional School Counseling, 4 (2), 113-119. Disponible en: http://ace. ucr.edu/people/nancy_guerra/nancy_pdfs/chil dren $\% 20$ who\%20get\%20victimized\%20at\%20 school\%20what\%20do\%20we\%20know.pdf

Haro-Solís, Israel \& García-Cabrero, Benilde (2014). Variables emocionales y sociomorales asociadas con el tipo de rol que asumen los alumnos y alumnas en el maltrato entre iguales. Apuntes de Psicología, 32 (1), 15-23. Disponible en: http:// www.apuntesdepsicologia.es/index.php/revista/ article/view/482/382

Haro-Solís, Israel; García-Cabrero, Benilde \& ReidlMartínez, Lucy María (2013). Experiencias de culpa y vergüenza en situaciones de maltrato entre iguales en secundaria. Revista Mexicana de Investigación Educativa, 18 (59), 1047-1075. Disponible en http://www.comie.org.mx/docu mentos/rmie/v18/n059/pdf/59002.pdf

Harris, Sandy; Petrie, Garth \& Willoughby, William (2002). Bullying among $9^{\text {th }}$ Graders: An Exploratory Study. NASSP Bulletin, 86 (630), 3-14.

Houndoumadi, Anastasia \& Pateraki, Lena (2001). BuIlying and Bullies in Greek Elementary Schools: Pupils' Attitudes and Teachers'/Parents' Awareness. Educational Review, 53 (1), 19-26. http:// dx.doi.org/10.1080/00131910120033619

Hymel, Shelley \& Swearer, Susan M. (2015). Four Decades of Research School Bullying. An Introduction. American Psychologist, 70 (4), 293-299. http://dx.doi.org/10.1037/a0038928

Jungert, Tomas; Piroddi, Barbara \& Thornberg, Robert (2016). Early Adolescents' Motivations to Defend Victims in School Bullying and their Perception of Student-Teacher Relationships: A Self-Determination Theory. Journal of Adolescence, 53, 75-90. http://dx.doi.org/10.1016/j.adolescence. 2016.09.001

Kahn, Jeffrey H.; Jones, Jayme L. \& Wieland, Amy L. (2012). Preservice Teachers' Coping Styles and their Responses to Bullying. Psychology in the Schools, 49, 784-793. DOI:10.1002/pits.21632

Kilian, Janet M.; Fish, Marian C. \& Maniago, Erica B. (2007). Making Schools Safe: A System-Wide School Intervention to Increase Student Prosocial Behavior and Enhance School Climate. Journal of Applied School Psychology, 23, 1-30. http:// dx.doi.org/10.1300/J370v23n01_01. Disponible en: https://psyc526final.wikispaces.com/ file/view/Making+Schools+Safe+a+system +w ide+school+intervention+to+increase+stude $\mathrm{nt}+$ prosocial+behaviors + and + enhance + scho ol+climate.pdf

Kochenderfer-Ladd, Becky \& Pelletier, Marie E. (2008). Teachers' Views and Belief about Bullying: Influences on Classroom Management Strategies and Students' Coping with Peer Victimization. Journal of School Psychology, 46 (4), 431-453. DOI: 10.1016/j.jsp.2007.07.005

Kokkinos, Constantinos \& Panayiotou, Georgia (2004). Predicting Bullying and Victimization among Early Adolescents: Associations with Disruptive Behavior Disorders. Aggressive Behavior, 30 (6), 520-533. http://dx.doi.org/10.1002/ab.20055

Loukas, Alexandra \& Murphy, Jonna L. (2007). Middle School Student Perceptions of School Climate: Examining Protective Functions on Subsequent Adjustment Problems. Journal of School Psychology, 45 (3), 293-309. http://dx.doi.org/10.1016/j. jsp.2006.10.001

Madrid-López, Esthela Jacqueline; Valdés-Cuervo, Ángel Alberto \& Vera-Noriega, José Ángel (2015). Diferencias en los estilos de afrontamiento de estudiantes víctimas y no involucrados en el buIlying. Ponencia presentada en el XIII Congreso Nacional de Investigación Educativa, Chihuahua, Consejo Mexicano de Investigación Educativa, COMIE. Disponible en: https://www.research gate.net/publication/289991832_Diferencias_ en_los_estilos_de_afrontamiento_de_estudian tes_victimas_y_no_involucrados_en_el_bullying

Mehta, Sharmila; Cornell, Dewey; Fan, Xitao \& Gregory, Anne (2013). Bullying Climate and School Engagement in Ninth-Grade Students. Journal of School Health, 83 (1), 45-52. DOI: 10.1111/j.17461561.2012.00746.x

Meltzer, Howard; Vostanis, Panos; Ford, Tamsin Jane; Bebbington, Paul E. \& Dennis, Michael Stuart (2011). Victims of Bullying in Childhood and Suicide Attempts in Adulthood. European Psychiatry, 26, 498-502. http://dx.doi.org/10.1016/j.eu rpsy.2010.11.006

México (2011). Acuerdo 592, por el que se establece la Articulación de la Educación Básica. Diario Oficial de la Federación, 19 de agosto de 2011. Disponible en: https://www.sep.gob.mx/work/ models/sep1/Resource/9721849d-666e-48b78433-0eec1247f1ab/a592.pdf

Meyer-Adams, Nancy \& Conner, Bradley T. (2008). School Violence: Bullying Behaviors and the Psychosocial School Environment in Middle Schools. Children \& Schools, 30 (4), 211-221. http://dx.doi.org/10.1093/cs/30.4.211

Muñoz-Abundez, Gustavo (2008). Violencia en México y en otros países. Comparaciones a partir de los resultados del Instituto Nacional para 
la Evaluación de la Educación. Revista Mexicana de Investigación Educativa, 13 (39), 1195-1228. Disponible en: http://www.redalyc.org/ pdf/140/14003908.pdf

Nation, Maury; Vieno, Alessio; Perkins, Douglas D. \& Santinello, Massimo (2008). Bullying in School and Adolescence Sense of Empowerment: An Analysis of Relationships with Parents, Friends, and Teachers. Journal of Community \& Applied Social Psychology, 18, 211-232. http://dx.doi. org/10.10 02/casp.921. Disponible en: http://onlinelibrary.wiley.com/doi/ 10.1002/casp.921/pdf

Nickerson, Amanda B. \& Martens, Matthew P. (2008). School Violence: Associations with Control, Security/Enforcement, Educational/Therapeutic Approaches, and Demographic Factors. School Psychology Review, 37 (2), 228-243. Disponible en: http://209-197-41-56.unassigned.ntelos. net/images/stories/library/Stennett_Psychology_Articles/School\%20 Violence\%20-\%20Associations\%20with\%20Control\%20Security-Enfor cement\%20Education-Therapeutic\%20Approaches\%20\%20Demogra phic\%20Factors.pdf

Nickerson, Amanda B. \& Spears, William H. (2007). Influences on Authoritarian and Educational/Therapeutic Approaches to School Violence Prevention. Journal of School Violence, 6 (4), 3-31. http://dx.doi.org/10.1300/ J202v06n04_02

O'Brennan, Lindsey M.; Bradshaw, Catherine \& Sawyer, Anne L. (2009). Examining Developmental Differences in the Social-Emotional Problems among Frequent Bullies, Victims, and Bully/Victims. Psychology in the Schools, 46 (2), 100-115. http://dx.doi.org/10.1002/pits.20357. Disponible en: https:// psyc525final.wikispaces.com/file/view/Examining+development+differe nces+in+the+social-emotional+problems+among +frequent+bullies, + victimes, +and+bully-victims.pdf

Olweus, Dan (2011). Bullying at School and Later Criminality: Finding from Three Swedish Community Samples of Males. Criminal Behaviour and Mental Health, 21 (2), 151-156. http://dx.doi.org/10.1002/cbm.806

Osher, David; Dwyer, Kevin P.; Jimerson, Shane R. \& Brown, Jacqueline A. (2012). Developing Safe, Supportive, and Effective Schools: Facilitating Student Success to Reduce School Violence. En Shane R. Jimerson, Amanda B. Nickerson, Matthew J. Mayer \& Michael J. Furlong (eds.). Handbook of School Violence and School Safety, 27-44. $2^{\text {nd }}$ ed. New York, New York: Taylor \& Francis. Disponible en: https://www.nycourts.gov/ip/justicefor children/PDF/NYS\%20Summit-Additional\%20Resources/P7\%20-\%20De veloping\%20Safe\%20Supportive\%20and\%20Effective\%20Schools.pdf

Rigby, Ken (2003). Consequences of Bullying in Schools. The Canadian Journal of Psychiatry, 48 (9), 583-590.

Salmivalli, Christina \& Nieminen, Eija (2002). Proactive and Reactive Aggression among School Bullies, Victims and Bully-Victims. Aggressive Behavior, 28 (1), 30-44. http://dx.doi.org/10.1002/ab.90004

Salmivalli, Christina \& Voeten, Marinus (2004). Connections between Attitudes, Group Norms, and Behaviour in Bullying Situations. International Journal of Behavioral Development, 28 (3), 246-258. http://dx.doi. org/10.1080/01650250344000488

Sangsue, J. \& Vorpe, G. (2004). Influences professionnelles et personnelles du climat scolaire chez les enseignants et les élèves. Psychologie du Travail et des Organizations, 10 (4), 341-354.

Shelton, Andrea J.; Owens, Emiel W. \& Song, Holim (2009). An Examination of Public School Safety Measures across Geographic Setting. Journal of School Health, 79 (1), 24-29. http://dx.doi.org/10.1111/j.1746-1561.2008.00370.x 
Stephens, Paul (2011). Preventing and Confronting School Bullying: A Comparative Study of Two National Programmes in Norway. British Educational Research Journal, 37 (3), 381-404. http:// dx.doi.org/10.1080/01411921003692868

Swearer, Susan M. \& Hymel, Shelley (2015). Understanding the Psychology of Bullying. Moving toward a Social-Ecological Diathesis-Stress Model. American Psychologist, 70 (4), 344-353. http://dx.doi.org/10.1037/a0038929. Disponible en: https://www.apa.org/pubs/journals/releases/ amp-a0038929.pdf

Swearer, Susan M.; Espegale, Dorothy L.; Koenig, Brian; Berry, Brandi; Collins, Adam \& Lembeck, Paige (2012). A Social-Ecological Model for Bullying Prevention and Intervention in Early Adolescence. En Shane R. Jimerson, Amanda B. Nickerson, Matthew J. Mayer \& Michael J. Furlong (eds.). Handbook of School Violence and School Safety, 333-355. $2^{\text {nd }}$ ed. New York, New York: Routledge.

Thomas, Duane E. \& Bierman, Karen L. (2006). The Impact of Classroom Aggression on the Development of Aggressive Behavior Problems in Children. Development Psychopathology, 18, 471-487. https://doi.org/10.1017/S0954579406060251. Disponible en: https://repository.upenn.edu/cgi/ viewcontent.cgi? article $=1178 \&$ context $=$ gse pubs

Trianes-Torres, María Victoria; Blanca-Mena, María José; Morena-Fernández, María Luisa de la; Infante-Cañete, Lidia \& Raya, S. (2006). Un cuestionario para evaluar el clima escolar del Centro Escolar. Psicothema, 18 (2), 272-277. Disponible en: http://www.psicothema.com/pdf/3209.pdf

Valdés-Cuervo, Ángel Alberto; Bautista-Hernández, Gildardo; Vera-Noriega, José Ángel \& HerreraMateos, Juan Carlos (2013). Variables que diferencian a estudiantes de secundaria con y $\sin$ reportes de bullying en la escuela. Psicología Iberoamericana, 21 (1), 32-41. Disponible en: http:// www.redalyc.org/pdf/1339/133929862005.pdf
Valdés-Cuervo, Ángel Alberto \& Carlos-Martínez, Ernesto Alonso (2014). Relación entre el autoconcepto social, el clima familiar y el clima escolar con el bullying en estudiantes de secundarias. Avances en Psicología Latinoamericana, 32 (3), 443-453. http://dx.doi.org/10.12804/ apl32.03.2014.07. Disponible en: http://revis tas.urosario.edu.co/index.php/apl/article/view/ apl32.03.2014.07

Valdés-Cuervo, Ángel Alberto; Martínez-Ferrer, Belén \& Carlos-Martínez, Ernesto Alonso (2018). El rol de las prácticas docentes en la prevención de la violencia escolar entre pares. Revista de Psicodidáctica, 23 (1), 33-38. Disponible en: https:// www.sciencedirect.com/science/article/pii/ S1136103417301041

Wang, Cixin; Berry, Brandi \& Swearer, Susan M. (2013). The Critical Role of School Climate in Effective Bullying Prevention. Theory Into Practice, 52 (4), 296-302. http://dx.doi.org/10.1080/00 405841.2013.829735. Disponible en: https:// www.researchgate.net/publication/272017571_ The_Critical_Role_of_School_Climate_in_Effec tive_Bullying_Prevention

Wei, Hsi-Sheng; Williams, James Herbert; Chen, Ji-Kang \& Chang, Hsiu-Yu (2010). The Effects of Individual Characteristic, Teacher Practice, and School Organizational Factor on Students' Bullying: A Multilevel Analysis of Public Middle Schools in Taiwan. Children and Youth Services Review, 32 (1), 137-143. https://doi.org/10.1016/j.child youth.2009.08.004

Whitted, Kathryn Suzanne \& Dupper, David R. (2005). Best Practices for Preventing or Reducing BuIlying in Schools. Child \& School, 27 (3), 167-175. http://dx.doi.org/10.1093/cs/27.3.167

Yoon, Jina S. \& Kerber, Karen (2003). Bullying: Elementary Teachers' Attitudes and Intervention Strategies. Research in Education, 69, 27-35. Disponible en: http://www.haghish.com/talk/anova/ Bullying.pdf 